\title{
Cationic Biocide Anchored Graphene Oxide-Based Membranes for Water Purification
}

\author{
PARESH KUMAR SAMANTARAY ${ }^{1}$ and SURYASARATHI BOSE ${ }^{2, *}$ \\ ${ }^{1}$ Centre for BioSystems Science and Engineering, Indian Institute of Science, Bangalore, India \\ ${ }^{2}$ Department of Materials Engineering, Indian Institute of Science, Bangalore, India
}

(Received on 12 September 2017; Revised on 18 November 2017; Accepted on January 2018)

\begin{abstract}
PVDF/PBSA [poly(vinylidene fluoride)/poly (butylene succinate-co-adipate)] phase inversion membranes were obtained using non-solvent induced phase separation and their porous hierarchical morphology were assessed using SEM. In order to render the membrane surface antibacterial, graphene oxide sheets were modified with a cationic biocide, trihexyltetradecylphosphonium chloride, and were subsequently grafted on the membrane surface through esterification. Successful grafting was confirmed by XPS and supported with NMR studies. A standard plate count method was used to assess the antimicrobial properties taking $E$. coli and $S$. aureus as model bacterial systems. To gain insight into the mechanism of bacterial inactivation, intracellular ROS (reactive oxygen species) generation was investigated on the microbial strains. The trans-membrane flux was measured using a cross-flow flux setup. For evaluating the long term efficacy of modified membranes; flux was also measured for different membranes both with pure water and bacterial feed. The membranes were excellent in retaining the flux in a long run after modifications. The anti-fouling performance was carried out using BSA (Bovine serum albumin) as a model biofoulant. The flux recovery ratio was significantly higher as compared to pristine membranes. Taken together, these modified membranes open new avenues in designing unique membranes for water purification.
\end{abstract}

Keywords: PVDF/PBSA Membranes; GO (Graphene oxide); Trans-membrane Flux; ROS; Anti-Fouling; antibacterial

\section{Introduction}

Earth being a blue planet is covered with $71 \%$ water, with only $2.5 \%$ of this water being source of fresh water (Petersen et al., 2017). About 2.6 billion of the world population are still deprived of fresh water and 3900 children die every day due to lack of safe drinking water sources (Qadir et al., 2017). These challenges have led to the development of new purification systems in order to meet the current needs. Out of all the options, polymeric membranes seek a variety of application in the domain of microfiltration, ultra filtration and reverse osmosis (Baker, 2000; Lonsdale, 1982; Staude, 1992). Out of many potential material alternatives, PVDF is sought extensively in the membrane industry due to its mechanical strength, thermal stability and chemical resistance (Liu et al., 2011). In the phase inversion process, transformation occurs from a homogenous polymer solution in a controlled manner from liquid to solid state (Kesting, 1985; Mulder, 2012). The transformation can be achieved by (a) thermally induced phase separation (TIPS) where the volatile solvent after evaporation escapes the system causing de-mixing and thereby phase separates; (b) precipitation from vapor phase; (c) solvent and nonsolvent exchange in the water bath which precipitates the homogenous state and leads to phase separation termed as immersion precipitation (IP) (Liu et al., 2011) PVDF membranes are fabricated majorly by this method because PVDF readily dissolves in common organic solvents (Liu et al., 2011).

Grafting the membranes with silver ion (Diagne et al., 2012; Mural et al., 2016b; Zhang et al., 2012; Zodrow et al., 2009), antibiotics (Ben-Sasson et al., 
2013; Xueli et al., 2013) or agents like anti-microbial polymer (Li et al., 2014; Liu et al., 2010), nano materials and enzymes (Mural et al., 2016a; Padmavathy et al., 2017; Pavlukhina et al., 2012; Schiffman and Elimelech, 2011; Zinadini et al., 2014) renders the anti-microbial properties to these membranes. However, these biocides are again divided on the basis of leaching and non-leaching biocides. Leaching biocides tethered to the membrane substrate deplete continuously with time and result in eventual loss of the antibacterial activity (Li et al., 2006). Hence, non-leaching agents are promising in the application and have good stable flux and antifouling properties. Recent literatures have already stated that graphene oxide (GO) exhibits anti-bacterial properties because of scissoring effect that causes irreversible damage to the microbial cells. (Mural et al., 2014; Mural et al., 2016a; Popa et al., 2003). In addition, cationic salts like phosphonium based compounds are also known to exhibit excellent antibacterial properties both towards $E$. coli and $S$. aureus (Kanazawa et al., 1993).

In this paper, we have designed a unique microporous PVDF/PBSA phase inversion membrane tethered with modified GO. Initially, the GO sheets were modified with a cationic biocide based on phosphonium salts and subsequently anchored onto the membrane surface. We have assessed the microbial viability of the membranes with two different strains by a standard plate count and the intracellular ROS generation was evaluated to gain insight into the mechanism of microbial killing.

\section{Materials and Methods}

\section{Experimental Section}

PVDF with $\mathrm{M}_{\mathrm{w}} 440000 \mathrm{~g} / \mathrm{mol}$ (KYNAR 761) was procured from Arkema. Bionolle 3001MD (PBSA) with $\mathrm{M}_{\mathrm{w}} 240000 \mathrm{~g} / \mathrm{mol}$ was gifted by SHOWA DENKO K.K Japan. Trihexyltetradecylphosphonium chloride, Bovine serum albumin, Luria Bertani broth, sodium hydroxide pellets, Sodium Chloride, 4(Dimethylamino) pyridine (DMAP), N,N'Dicyclohexylcarbodiimide were procured from Sigma Aldrich. Nutrient Agar was purchased from Fluka. Ethanol was obtained from SD Fine Chemicals Limited. GO was procured from Bottom Up Technologies Corporation.

\section{Membrane Preparation}

The doped solution for the membrane was made by dissolving $0.64 \mathrm{~g}$ of PVDF in $4 \mathrm{~mL}$ of DMF. To this, $0.16 \mathrm{~g}$ of PBSA was added and was mechanical stirred at $50^{\circ} \mathrm{C}$ till a homogenous solution was obtained. The solution was kept to rest for an hour and then casted using a doctor blade on a glass substrate and submerged in water bath maintained at $4^{\circ} \mathrm{C}$ to induce precipitation. The membrane was then washed thoroughly with water and dried in vacuum oven at $80^{\circ} \mathrm{C}$ overnight.

\section{Graphene Oxide Modification with Phosphonium Chloride}

$200 \mathrm{mg}$ graphene oxide was dispersed in $100 \mathrm{~mL}$ solution of water and ethanol (1:4 ratio of water and ethanol) using bath sonication for $30 \mathrm{~min}$. To this solution, $1 \mathrm{~g}$ trihexyltetradecylphosphonium chloride was added. The reaction was mechanically stirred for $24 \mathrm{~h}$ at $50^{\circ} \mathrm{C}$. The $\mathrm{GO}$ obtained was then washed with 1:1 ethanol water solution to remove the unreacted compound, centrifuged and vacuum filtered to obtain phosphonium anchored GO. The obtained phosphonium anchored $\mathrm{GO}$ was dried in vacuum oven at $25^{\circ} \mathrm{C}$ overnight.

\section{Membrane Modifications with Phosphonium Anchored GO}

$100 \mathrm{mg}$ phosphonium anchored graphene oxide was dispersed in $100 \mathrm{~mL}$ solution of water and ethanol (1:1 ratio of water and ethanol) using bath sonication for $30 \mathrm{~min}$. To this dispersion, $1 \mathrm{mmol}$ of DCC and DMAP were added and the pristine PVDF/PBSA membrane was immersed to carry out the esterification reaction between $-\mathrm{COOH}$ group of PBSA and $-\mathrm{OH}$ group of $\mathrm{GO}$. The reaction temperature was maintained at $0^{\circ} \mathrm{C}$ for $2 \mathrm{~h}$ with constant stirring. Then, the reaction mixture was stirred at $25^{\circ} \mathrm{C}$ for $1 \mathrm{~h}$.

\section{Characterization of Phosphonium Anchored GO}

The phosphonium anchored GO was characterized by ${ }^{31} \mathrm{P}$ NMR using JOEL ECX 500 High Resolution NMR Spectrometer at $500 \mathrm{MHz}$ and Raman spectroscopy was done using LabRAM HR. 


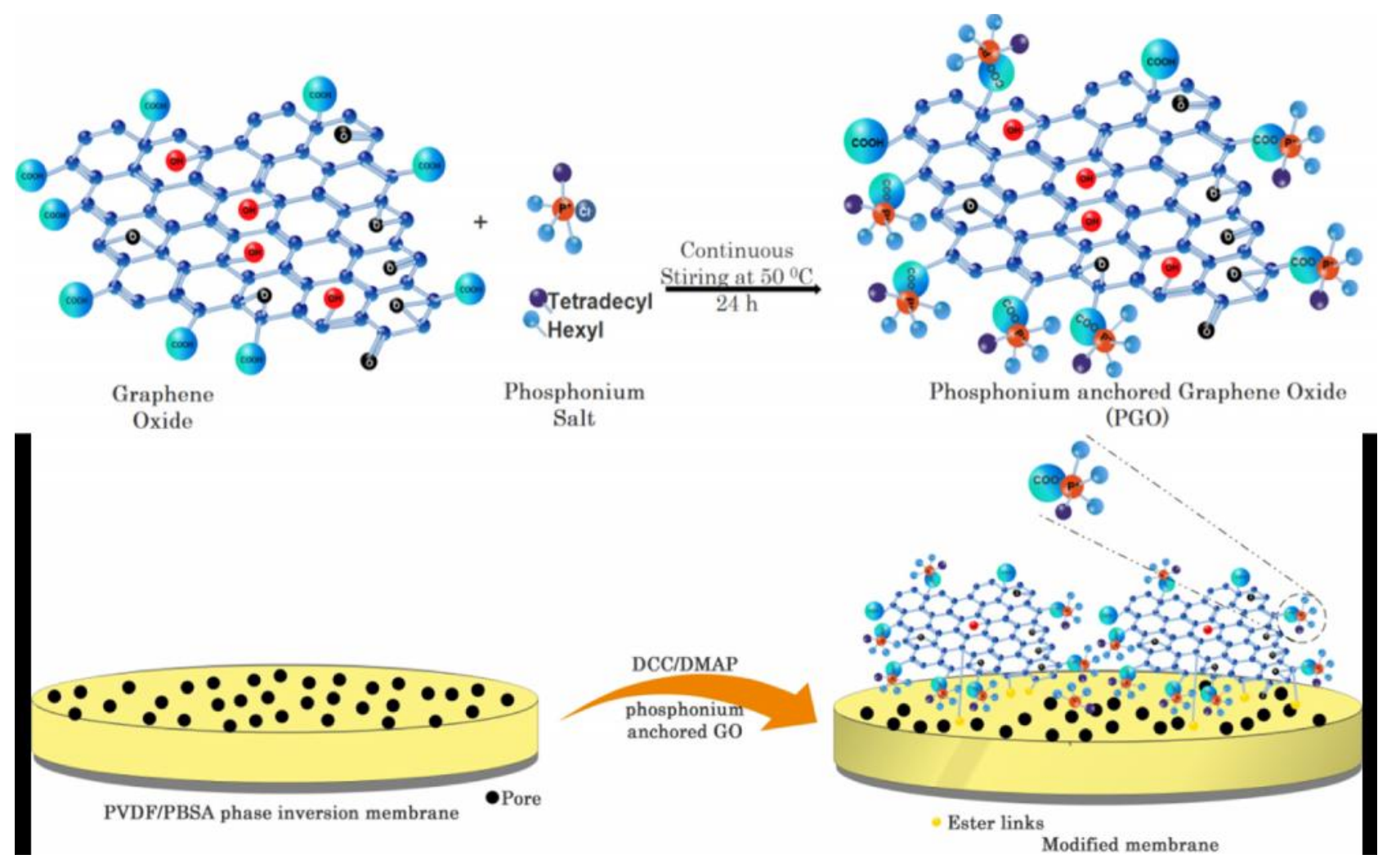

Scheme 1: Surface modification of PVDF/PBSA phase inversion membrane

\section{Characterization of the Membrane}

The phosphonium anchored GO modified PVDF/ PBSA membrane was characterized by ${ }^{31} \mathrm{P}$ NMR using JOEL ECX 500 High Resolution NMR Spectrometer at $500 \mathrm{MHz}$ and $\mathrm{X}$-ray photoelectron spectroscopy on Axis-Ultra using $\mathrm{Al}$ as the monochromatic source $(1.486 \mathrm{keV})$.

\section{Surface Morphology, Membrane Uptake and Porosity}

Hydrophilicity/hydrophobicity of the surface was assessed by contact angle. Change in contact angle from hydrophobic to hydrophilic makes it affine to the water molecules. Hence, a contact angle test with water was performed on all samples. To understand the bulk hydrophilicity of a membrane its water uptake capacity is determined. 10 membranes were cut into equal area of $0.63 \mathrm{~cm}^{2}$ and were vacuum-dried and weighed. These membranes were soaked in distilled water for $24 \mathrm{~h}$ and again weighed. The percentage uptake of water was calculated as per Hebbar et al. (Hebbar et al., 2016) :

$$
\% \text { Uptake }=\left(\frac{\mathrm{W} w-\mathrm{W} d}{\mathrm{~W} w}\right) \times 100
$$

Where, $W w$ and $\mathrm{W} d$ are the weights postswelling for $24 \mathrm{~h}$ and pre-swelling conditions respectively.

The weight of the wet membrane $(\mathrm{W} w)$ was noted, after wiping it using a filter paper. The membrane was placed in an air circulating oven at $75^{\circ} \mathrm{C}$ for $24 \mathrm{~h}$ and the dry weight $(\mathrm{W} d)$ was then measured.

The porosity $\varepsilon(\%)$ was found out using the equation:

$$
\varepsilon(\%)\left(\frac{W w-W d}{W \times l \times \rho}\right) \times 100
$$

Here, $A, l$ and $\rho$ denotes the membrane area, thickness of the membrane and density of pure water respectively. 


\section{Membrane Performance and Antifouling Studies}

The flux of pure water was determined by in-house cross flow setup. The token membranes were fixed into test cell of the setup and were subjected to different trans-membrane pressure using a HPLC pump. Triplicates of each were compacted at a pressure for $30 \mathrm{~min}$ before the experiment. The pure water flux, $\left(J_{w}\right)\left(\mathrm{L} \mathrm{m}^{-2} \mathrm{~h}^{-1}\right)$ was calculated by:

$$
J_{w}=\frac{V}{A \times t}
$$

where, $V$ is the permeate volume, $A$ is the area of membrane and $t$ is the elution time.

Membrane resistance $\left(R_{m}\right)$ was determined by the inverse of the slope of the plot of trans-membrane flux $\left(J_{w}\right)$ versus the trans-membrane pressure $(\Delta \mathrm{P})$. To assess the long term performance, pure water flux data for 30 days was obtained at $0.2 \mathrm{MPa}$ for the membranes.

Organic foulants generally block the open channels and get physically adsorb on the surface. This increases the membrane resistance thereby reducing the shelf life of the membranes. Hence, reversible antifouling effect is needed to be studied. It is reported that surfaces which are reversible to antifouling effect are suitable for membrane application (Zhao and He, 2015). For reversibility evaluation a back flushing cycle is done after the fouling material has been charged and filtered. The ratio of flux post back flushing to the initial pure water flux defines the membrane reversibility. For this, Bovine serum Albumin (BSA) was chosen and the process was carried out as follows (Zhao and He, 2015). (a) stable pure water flux $\left(J_{w}\right)$ by pure water filtration, (b) stable flux $\left(J_{P}\right)$ obtained after input is changed to organic foulant (BSA concentration at 1 $\mathrm{g} / \mathrm{L}$ ). (c) Second cycle of pure water flux $(J)$ after a back flush cycle of 15 min with phosphate buffer saline solution. Membrane triplicates were taken to perform the entire experiment. In this test, the flux recovery ratio (FRR), irreversible flux decline ratio (IFR) and relative fouled flux ratio (RFR) help to understand the antifouling. The FRR, IFR and RFR are given by:

$$
\operatorname{FRR}(\%)=\left(\frac{J}{J_{w}}\right) \times 100
$$

$$
\begin{aligned}
& \operatorname{IFR}(\%)=100-\operatorname{FRR}(\%) \\
& \operatorname{RFR}(\%)=\left(\frac{J_{p}}{J_{w}}\right) \times 100
\end{aligned}
$$

\section{Antibacterial Studies}

The antibacterial assessment was performed by taking ATCC25922 Escherichia coli (Gram-negative bacteria) and ATCC25923 Staphylococcus aureus (Gram-positive bacteria) as model bacterial strains. The strains were sub-cultured in LB broth media at $37^{\circ} \mathrm{C}$ in a shaking incubator and harvested. Bacterial pellets obtained by centrifugation were washed with PBS (phosphate buffered saline) and were resuspended for the experiment.

\section{Standard Plate Count}

The membranes were cut into $4.5 \mathrm{~mm}$ disks and were put in 96 well-plate. $100 \mu \mathrm{L}$ of E. coli and S. aureus PBS suspended culture was added to well. The wellplate was incubated in a shaking incubator for $30 \mathrm{~min}$, $1 \mathrm{~h}$ and $2 \mathrm{~h}$. The suspension was collected and $100 \mu \mathrm{L}$ of 7-fold serial dilutions were cultured in nutrient agar using spreader technique and incubated at $37^{\circ} \mathrm{C}$ for $24 \mathrm{~h}$. The colonies were then calculated after $24 \mathrm{~h}$ incubation.

\section{Intracellular ROS Generation}

Whenever anti-microbial agents come in contact with the microbial cell surface, it induces membrane stress by physical contact. This induction generates intracellular reactive oxygen species which inhibits bacterial growth. To evaluate the ROS generated by the microbes due to contact, DCFH-DA (dichlorodihydrofluorescein diacetate) dye was used. DCFH-DA is non-fluorescent in nature and penetrates into the cell membrane of the microbes. In the presence of cellular esterase upon oxidation, it is converted to DCF which emits fluorescent green light. The membranes of $4.5 \mathrm{~mm}$ disks were incubated with $100 \mu \mathrm{L}$ of $E$. coli and $S$. aureus culture respectively for $30 \mathrm{~min}, 1 \mathrm{~h}$ and $2 \mathrm{~h}$ in a 96 well-plate in a shaking incubator. $50 \mu \mathrm{L}$ of the dye was added to the system after the incubation time and was kept in dark for $30 \mathrm{~min}$. The measurements were then taken at the excitation wavelength of $485 \mathrm{~nm}$ and emission at 528 $\mathrm{nm}$. 


\section{Results and Discussion}

\section{Characterization of Phosphonium Anchored GO}

The chemical shift for Trihexyltetradecylphosphonium chloride was found to be $33.33 \mathrm{ppm}$ in ${ }^{31} \mathrm{P}$ NMR. The modified GO however showed a shift of $37.95 \mathrm{ppm}$ indicating that the GO has been successfully modified. This was supported by Raman spectroscopy of unmodified GO and modified GO. The D band was found in $1347 \mathrm{~cm}^{-1}$ and $\mathrm{G}$ band at $1595 \mathrm{~cm}^{-1}$ for un-modified GO. The D/G ratio was found to be 1.06 . Whereas after phosphonium salt was anchored to the GO, there was a reduction in the $\mathrm{D}$ band indicating that the $\mathrm{COOH}$ groups present in defect edges of the GO have interacted with Trihexyltetradecylphosphonium chloride forming phosphonium carboxylate. The D/G ratio of the modified GO was found to be 0.87 . Fig. 1 represents the Raman spectra of GO modification.

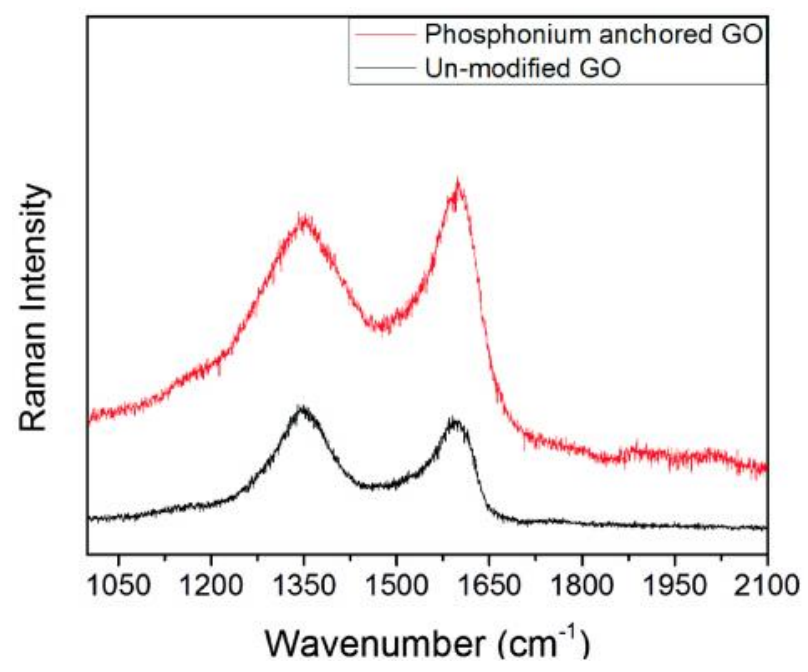

Fig. 1: Raman spectra of unmodified and modified GO

\section{Characterization of Membrane}

To support the grafting process, XPS was also because it is known to be a surface sensitive characterization tool. Figure 2(A) shows the survey scan of the samples. Figure 2(B) shows the C1s spectrum of Neat PVDF/PBSA membrane. C-C binding energy at $284.8 \mathrm{eV}$ whereas $\mathrm{C}-\mathrm{O}$ bond of PBSA showed a binding energy of $286.2 \mathrm{eV}$ and $\mathrm{C}=\mathrm{O}$ of PBSA at $288.6 \mathrm{eV}$. However, to show presence of phosphonium anchored GO, C1s spectrum of $\mathrm{PVDF} /$ PBSA membrane with the modification was recorded as seen in Fig. 2(C). Apart from the other bonds $\mathrm{C}=\mathrm{C}$ bond due to presence of GO in the membrane surface was also captured at $283.3 \mathrm{eV}$. Moreover, $\mathrm{P}_{2 \mathrm{p}}$ spectrum of the same (Fig. 2(D) shows a binding energy of $133.4 \mathrm{eV}$ confirming the successful grafting of modified GO on the surface.

\section{Surface Morphology, Membrane Uptake and Porosity}

Figure 3(A) shows the surface morphology of the modified membrane. Asymmetric pores in the range of 300-600 nm were found by using image J analysis. Figure 3(b) shows the contact angles of different samples. For un-modified membranes, the contact angle was found to be $75 \pm 3^{\circ}$ whereas with phosphonium modified GO grafted membranes, it was found to be $47 \pm 4^{\circ}$. It is clear that the hydrophilicity of the membrane has enhanced upon modification.

The uptake (in \%) was assessed to describe the hydrophilicity of the membranes. Equation (1) was used to calculate the same. It is well stated in literature (Ganesh et al., 2013) that water uptake capacity directly depends on the available hydrophilic sites and the morphology of the membrane. The uptake percent of the pristine PVDF/PBSA membrane and phosphonium anchored $\mathrm{GO}$ grafted membrane were found out to be $54.2 \pm 4.8 \%, 65.8 \pm 3.6 \%$ respectively. From this, it is clearly evident that the uptake has increased with graphene based modification onto PVDF/PBSA membranes. For the porosity $(\varepsilon, \%)$ calculation equation (2) was adopted. The porosity $(\varepsilon, \%)$ for pristine PVDF/PBSA membrane and phosphonium anchored GO grafted membrane was found to be $44 \pm 3.2 \%$ and $45 \pm 3.7 \%$ respectively, which is comparable, supporting the fact that the current surface modification didn't alter the porosity of membranes.

\section{Membrane Performance and Antifouling Studies}

The pure water flux for all the membranes were evaluated by an in-house designed cross flow setup as shown in Fig. 4(A). Trans-membrane flux of the pristine PVDF/PBSA membrane at $0.2 \mathrm{MPa}$ pressure was found to be $1345.1 \pm 35.3 \mathrm{Lm}^{-2} \mathrm{~h}^{-1}$ whereas, for phosphonium anchored GO grafted membrane at the same pressure, flux of $1446.5 \pm 38.3 \mathrm{Lm}^{-2} \mathrm{~h}^{-1}$ was obtained (Fig. 4(B). This suggests that the modifications which made the surface hydrophilic increased the affinity of water towards the membrane 

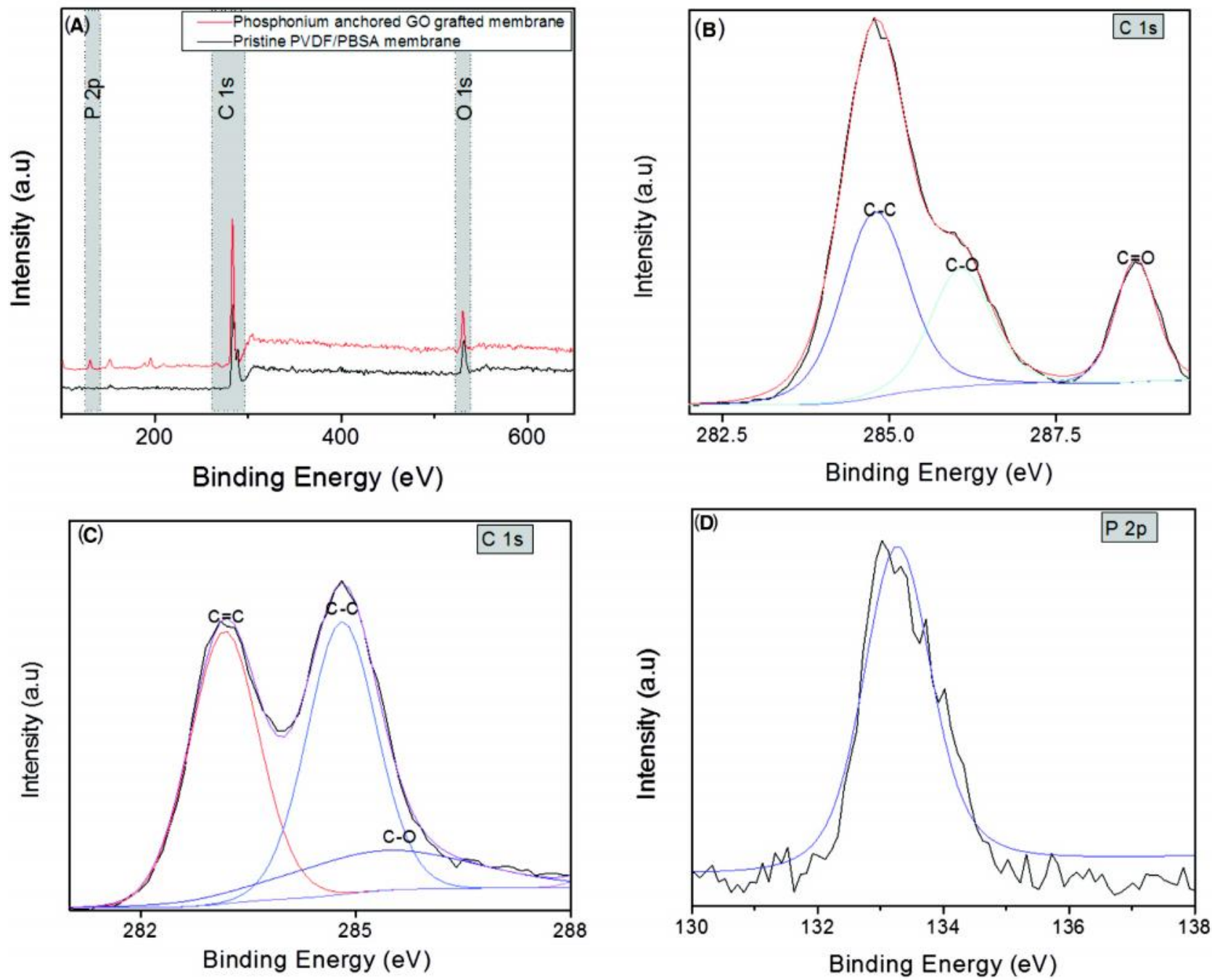

Fig. 2: (A) Survey scan of all modifications, (B) C 1s spectrum of pristine PVDF/PBSA, (C) C 1s spectrum of phosphonium anchored GO grafted PVDF/PBSA and (D) P 2p spectrum of phosphonium anchored GO grafted PVDF/PBSA
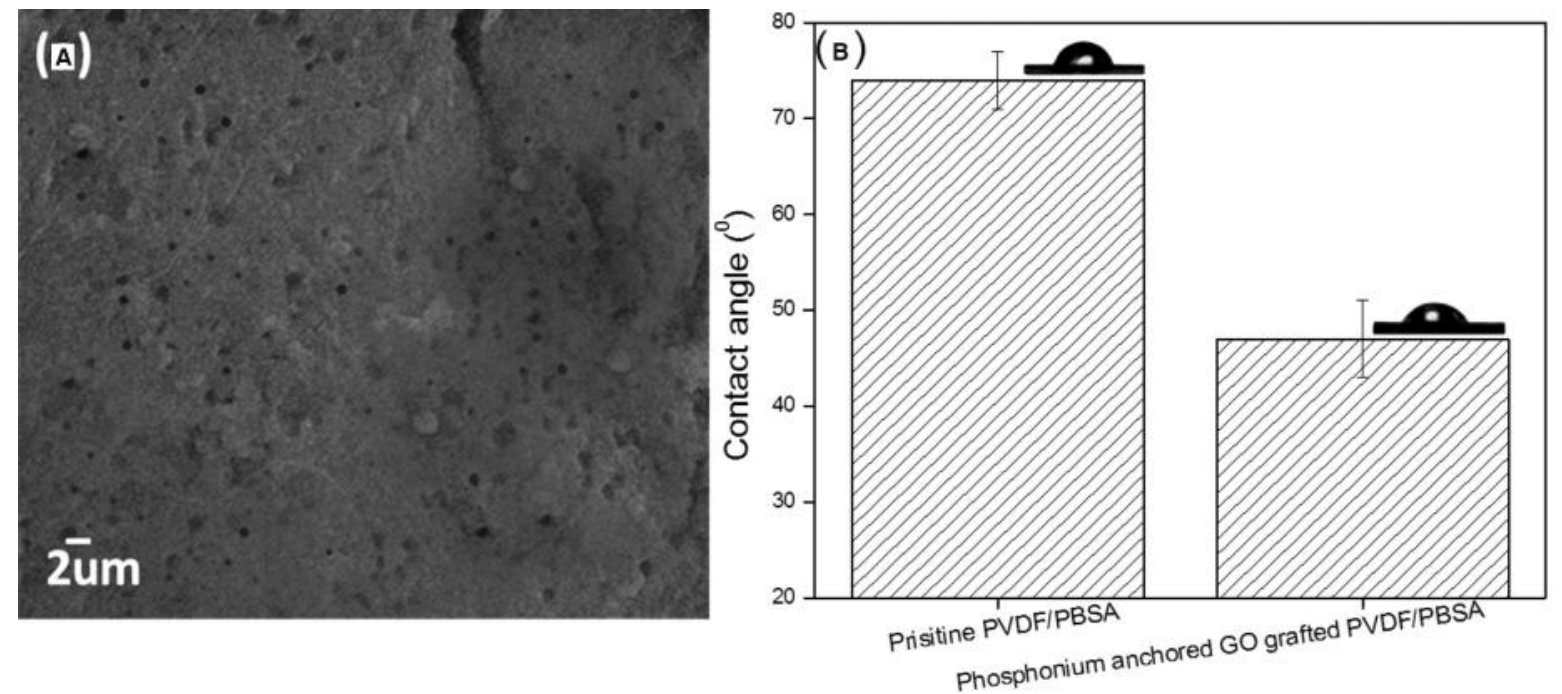

Fig. 3: (A) Surface morphology of the modified membrane, (B) Water contact angle for all the membrane samples 


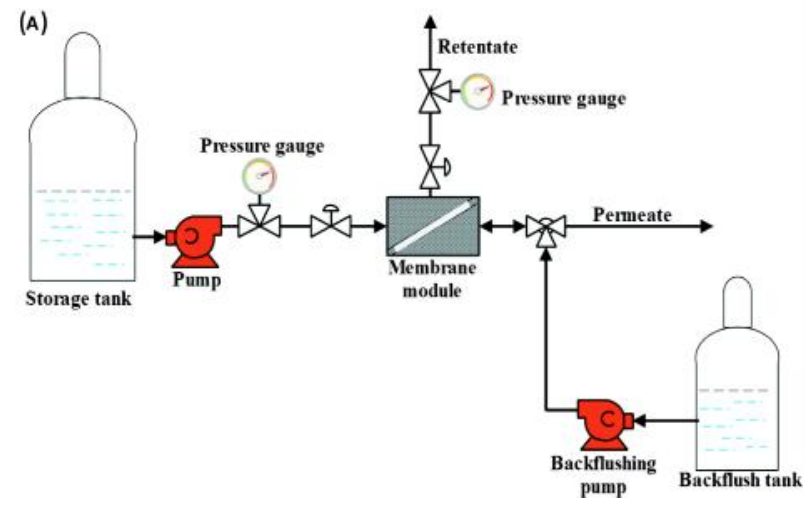

for phosphonium leeching using ICP-OES measurement and it was found that there was no significant leeching of the phosphonium salt anchored on the GO even after 15 days of continuous purification $(<0.01 \mathrm{ppm})$ The amount of leeching which was observed was within the WHO water norms.

A flux experiment at $0.2 \mathrm{MPa}$ was also carried out to check effectiveness of membranes on a long run. Fig. 4(C) shows the trans-membrane flux as a function of time. While the pristine membrane attained saturation after $600 \mathrm{~h}\left(857 \mathrm{Lm}^{-2} \mathrm{~h}^{-1}\right)$, phosphonium
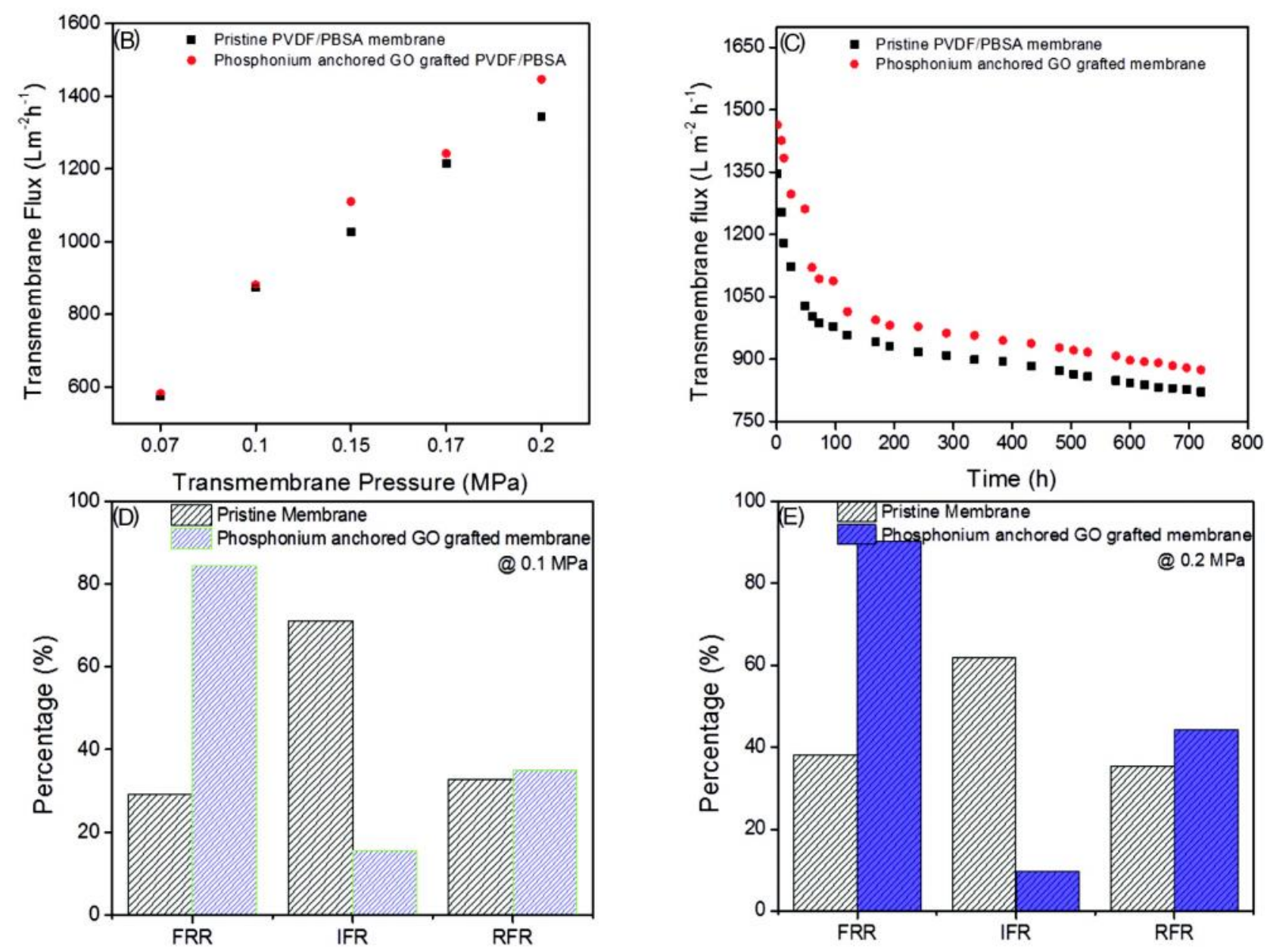

Fig. 4: (A) Schematics of flux setup, (B) pure water flux of all the membranes, (C) flux study as a function of time for all the membranes, (D) and (E) show the antifouling studies with BSA as foulant at $0.1 \mathrm{MPa}$ and $0.2 \mathrm{MPa}$ respectively

and led to more flux, which is supported by membrane hydraulic resistance $(\mathrm{Rm})$ evaluated from the inverse of the slope of Fig. 4(B). For un-modified membrane, $\mathrm{Rm}$ was $178 \mathrm{~Pa} \mathrm{~L}^{-1} \mathrm{~m}^{2} \mathrm{~h}$ and for phosphonium anchored GO grafted membrane it was observed to be $156 \mathrm{~Pa} \mathrm{~L}^{-1} \mathrm{~m}^{2} \mathrm{~h}$. The permeate was also analyzed anchored GO grafted membrane attained 917 $\mathrm{Lm}^{-2} \mathrm{~h}^{-1}$.

The dynamic membrane fouling test was carried out using BSA as a model biofoulant. This test helps in studying the antifouling property of a membrane. The FRR value for phosphonium anchored GO grafted 

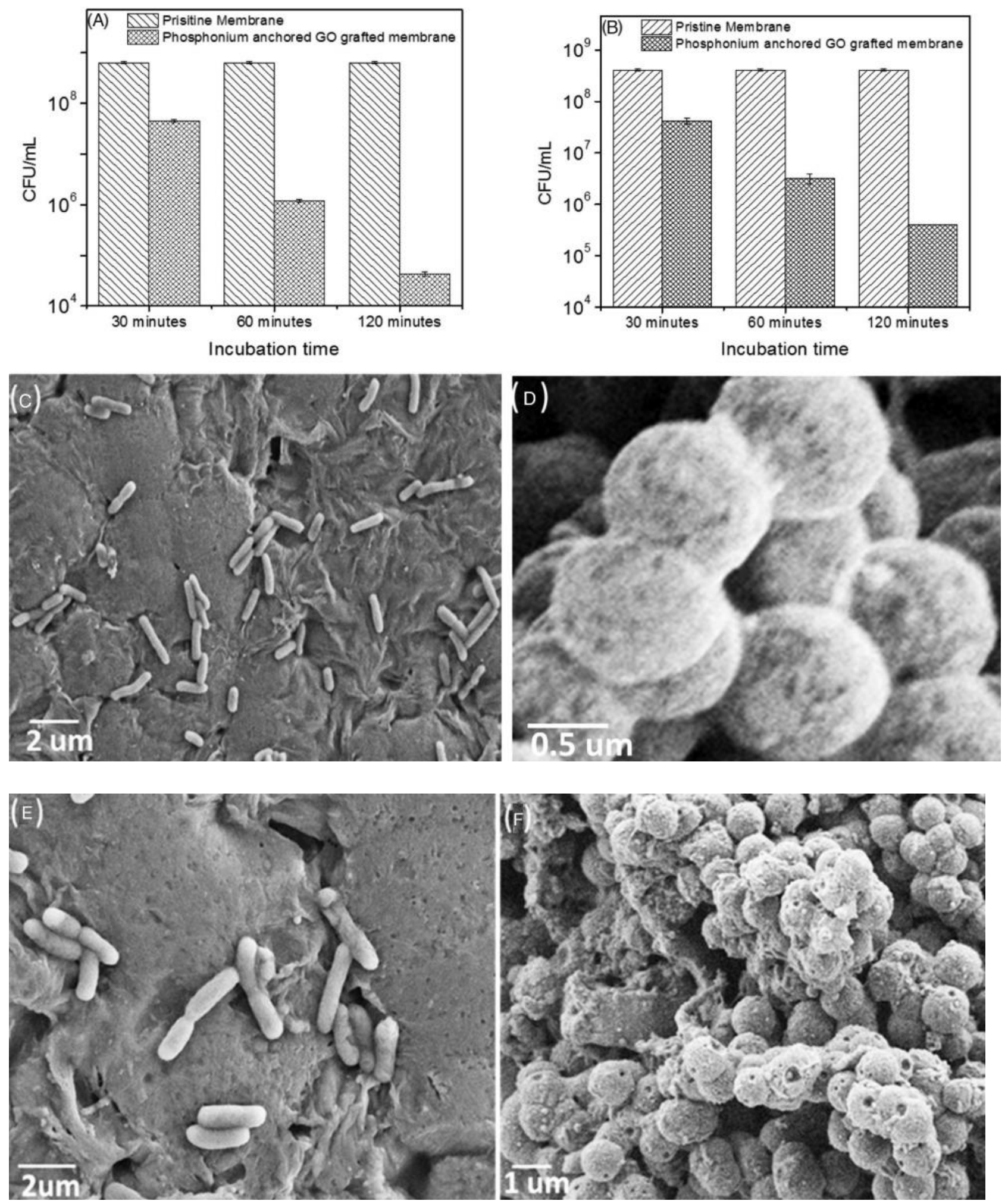

Fig. 5: (A) and (B) show the standard plate count results with 3 different incubation time, taking $E$. coli and $S$. aureus as model bacteria, (C) and (D) show the SEM image of pristine membrane after $2 \mathrm{~h}$ of bacterial incubation with $E$. coli and $S$. aureus respectively, (E) and (F) shows the phosphonium anchored GO grafted membrane after $2 \mathrm{~h}$ of bacterial incubation with $E$. coli and $S$. aureus respectively. The arrow mark shows irreversible bacterial cell damage and punctured bacteria 
membrane was $90.3 \pm 0.2 \%$ at $0.2 \mathrm{MPa}$ and $84.5 \pm$ $0.3 \%$ at $0.1 \mathrm{MPa}$. High IFR value of a membrane infers that it is more susceptible to bio-fouling due to its hydrophobicity. It can be clearly seen from Fig. 4(D) and (E) that pristine PVDF/PBSA membranes are more prone to fouling as compared to the phosphonium modifications.

\section{Antibacterial Studies}

To evaluate the antimicrobial effectiveness ATCC25922 Escherichia coli (Gram-negative bacteria) and ATCC25923 Staphylococcus aureus (Gram-positive bacteria) model bacterial strains have been chosen in this work.

\section{Standard Plate Count}

The antimicrobial action of phosphonium anchored GO grafted membrane was assessed by standard plate count with both the bacterial strains. As seen from Fig. 5(A), 1-log fold reduction was seen within $30 \mathrm{~min}$ of incubation with $E$. coli. After 2 h, the $E$. coli incubated phosphonium anchored GO grafted membrane showed a 4-fold bacterial reduction. While for $S$. aureus, 1-log fold reduction was seen after 30 min. After $2 \mathrm{~h}$ of incubation, 3-fold reduction was seen (Fig. 5(B). Furthermore, the incubated samples of $2 \mathrm{~h}$ incubation were fixed with $3.7 \%$ formaldehyde and visualized using SEM. (Fig. 5(C)-(F))

\section{Intracellular ROS Generation}

Intracellular reactive oxygen species (ROS) are the

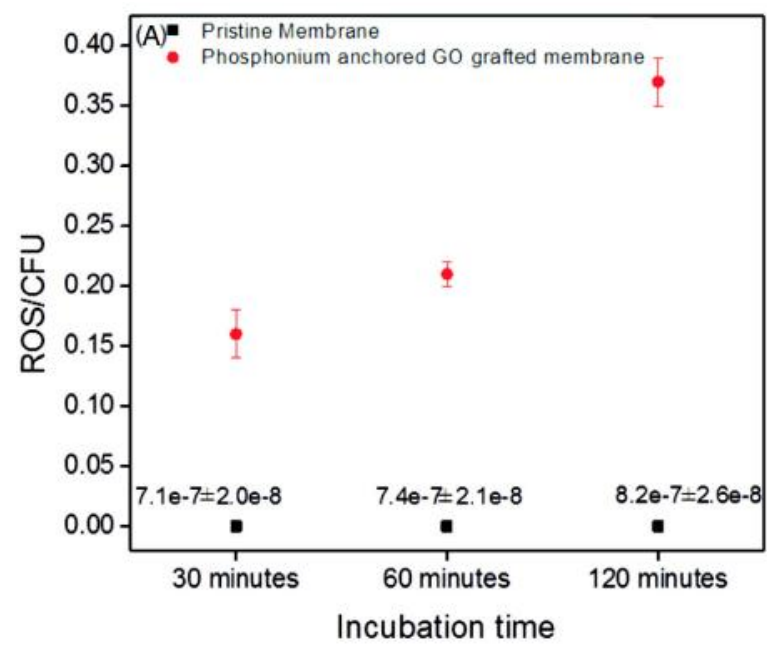

result of physical stresses induced by contact of antimicrobial compound with the microbial cell. These ROS inhibits bacterial growth. To study the ROS generated by microbes, DCFH-DA (dichlorodihydrofluorescein diacetate) dye was used. DCFH-DA is a non-fluorescent dye which penetrates the cell membrane of the microbes. In the presence of cellular esterase, they oxidize and get converted to DCF emitting fluorescent green light. The ROS which was obtained in counts per second was then divided by the live cells in each to obtain ROS/CFU value. It is clear from the Fig. 6(A) and (B) for E. coli and $S$. aureus respectively that phosphonium anchored GO induced significant ROS by contact as compared to pristine membranes.

Cationic biocide like $\mathrm{P}^{+}$cation has strong ionic charge which interacts with the outer membrane of bacterial cell and induces ROS. But, the biocidal action of this biocide can be enhanced with the combination of GO based modification. It can be concluded from these tests that when membranes tethered with this modification come in contact with the microbial cells, primary response by these cells is generation of ROS. The decimal reduction is mediated by ROS. But, when the incubation period is sufficient enough, $(2 \mathrm{~h}$ in this case) the $\mathrm{P}^{+}$cation present induces irreversible cell damage (evident from SEM). This work hypothesizes that the antimicrobial effects seen are the combination of the ROS and direct contact killing by the bacteria. The driving force in the lower time points is only ROS while in later stage, it is majorly driven by ROS and the compound itself.

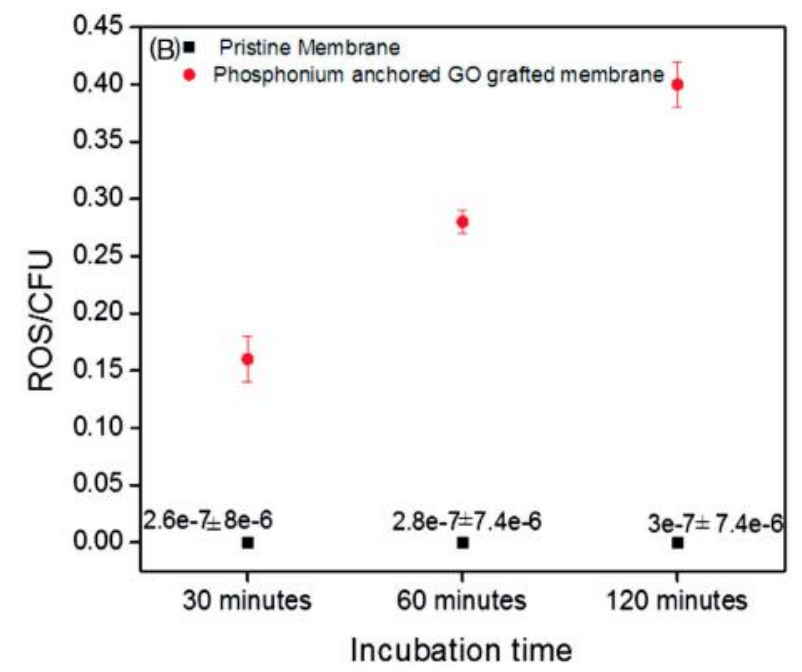

Fig. 6: (A) and (B) ROS/CFU for different membranes with E. coli and S. aureus as model organism 


\section{Conclusions}

In this work, micro porous PVDF/PBSA membranes were designed and tethered with phosphonium anchored graphene oxide. The anti-microbial action of the modified membranes was analyzed by standard plate count and the intracellular reactive oxygen species was studied by DCFH-DA. 1-log reduction for both the bacterial strains was noted after $30 \mathrm{~min}$ of incubation time. In addition to the antimicrobial properties, the modified membranes exhibited good fouling resistance against bovine serum albumin. Also, membrane flux of phosphonium anchored GO membranes was found to be $1446.5 \pm 38.3 \mathrm{Lm}^{-2} \mathrm{~h}^{-1}$ at $0.2 \mathrm{MPa}$ whereas for pristine PVDF/PBSA membrane at $0.2 \mathrm{MPa}$ pressure a flux value of 1345.1 $\pm 35.3 \mathrm{Lm}^{-2} \mathrm{~h}^{-1}$ was obtained. The flux retention

\section{References}

Baker R W (2000) Membrane technology. Wiley Online Library

Ben-Sasson M, Zodrow K R, Genggeng Q, Kang Y, Giannelis E $P$, et al. (2013) Surface functionalization of thin-film composite membranes with copper nanoparticles for antimicrobial surface properties Environ Sci Technol $\mathbf{4 8}$ 384-393

Diagne F, Malaisamy R, Boddie V, Holbrook R D, Eribo B, et al. (2012) Polyelectrolyte and silver nanoparticle modification of microfiltration membranes to mitigate organic and bacterial fouling Environ Sci Technol 46 4025-4033

Ganesh B, Isloor A M and Ismail A F (2013) Enhanced hydrophilicity and salt rejection study of graphene oxidepolysulfone mixed matrix membrane Desalination 313 199207

Hebbar R S, Isloor A M, Ananda K and Ismail A (2016) Fabrication of polydopamine functionalized halloysite nanotube/ polyetherimide membranes for heavy metal removal $J$ Mater Chem A 4 764-774

Kanazawa A, Ikeda T and Endo T (1993) Novel polycationic biocides: synthesis and antibacterial activity of polymeric phosphonium salts J Polym Sci Part A: Polym Chem 31 335-343

Kesting R E (1985) Phase inversion membranes. In: ACS symposium series. Oxford University Press, 131-164

Li X, Cai T and Chung T-S (2014) Anti-fouling behavior of hyperbranched polyglycerol-grafted poly (ether sulfone) hollow fiber membranes for osmotic power generation ratio for phosphonium anchored $\mathrm{GO}$ grafted membrane was $90.3 \pm 0.2 \%$ at $0.2 \mathrm{MPa}$ and $84.5 \pm$ $0.3 \%$ at $0.1 \mathrm{MPa}$. Apart from this, these modified membranes were stable on a long run experiment for 30 days with pure water as input. These membranes are hence found to be suitable for water purification application.

\section{Acknowledgement}

The authors would like to acknowledge the Department of Science and Technology for their financial support and CenSE IISc for various characterization facilities. The authors would also like to acknowledge Dr. Kaushik Chatterjee, Materials Engineering Department for extending the facilities for bacterial studies.

\section{Environ Sci Technol 48 9898-9907}

Li Z, Lee D, Sheng X, Cohen R E and Rubner M F (2006) Twolevel antibacterial coating with both release-killing and contact-killing capabilities Langmuir 22 9820-9823

Liu C, Zhang D, He Y, Zhao X and Bai R (2010) Modification of membrane surface for anti-biofouling performance: Effect of anti-adhesion and anti-bacteria approaches $J \mathrm{Membr}$ Sci 346 121-130

Liu F, Hashim N A, Liu Y, Abed M M and Li K (2011) Progress in the production and modification of PVDF membranes $J$ Membr Sci 375 1-27

Lonsdale H (1982) The growth of membrane technology J Membr Sci 10 81-181

Mulder J (2012) Basic principles of membrane technology. Springer Science \& Business Media

Mural P K S, Banerjee A, Rana M S, Shukla A, Padmanabhan B, et al. (2014) Polyolefin based antibacterial membranes derived from PE/PEO blends compatibilized with amine terminated graphene oxide and maleated PE J Mater Chem A 2 17635-17648

Mural P K S, Jain S, Kumar S, Madras G and Bose S (2016a) Unimpeded permeation of water through biocidal graphene oxide sheets anchored on to $3 \mathrm{D}$ porous polyolefinic membranes Nanoscale 8 8048-8057

Mural P K S, Jain S, Madras G and Bose S (2016b) Improving antifouling ability by site-specific silver decoration on polyethylene ionomer membranes for water remediation: assessed using 3D micro computed tomography, water 
flux and antibacterial studies RSC Adv.s 6 88057-88065

Padmavathy N, Samantaray P, D G L, Madras G and Bose S (2017) Selective Cleavage of Polyphosphoester in Crosslinked Copper based Nanogels: Enhanced Antibacterial Performance Through Controlled release of Copper Nanoscale 10.1039/C1037NR02446K

Pavlukhina S V, Kaplan J B, Xu L, Chang W, Yu X, et al. (2012) Noneluting enzymatic antibiofilm coatings ACS Appl Mater Interfaces 4 4708-4716

Petersen L, Heynen M and Pellicciotti F (2017) Freshwater Resources: Past, Present, Future The International Encyclopedia of Geography

Popa A, Davidescu C, Trif R, Ilia G, Iliescu S, et al. (2003) Study of quaternary 'onium'salts grafted on polymers: Antibacterial activity of quaternary phosphonium salts grafted on 'gel-type'styrene-divinylbenzene copolymers React Funct Polym.s 55 151-158

Qadir D, Mukhtar H and Keong L K (2017) Mixed Matrix Membranes for Water Purification Applications Sep Purif $\operatorname{Rev} 46$ 62-80

Schiffman J D and Elimelech M (2011) Antibacterial activity of electrospun polymer mats with incorporated narrow diameter single-walled carbon nanotubes ACS Appl Mater Interfaces A 3 462-468
Staude E (1992) Marcel Mulder: Basic Principles of Membrane Technology, Kluwer Academic Publishers, Dordrecht, Boston, London, 1991, ISBN 079230978 2, 363 Seiten, Preis: DM 200, Berichte der Bunsengesellschaft für physikalische Chemie 96 741-742

Xueli G, Haizeng W, Jian W, Xing H and Congjie G (2013) Surfacemodified PSf UF membrane by UV-assisted graft polymerization of capsaicin derivative moiety for fouling and bacterial resistance J Membr Sci 445 146-155

Zhang M, Zhang K, De Gusseme B and Verstraete W (2012) Biogenic silver nanoparticles (bio-Ag 0) decrease biofouling of bio-Ag 0/PES nanocomposite membranes Water Res 46 2077-2087

Zhao X and He C (2015) Efficient Preparation of Super Antifouling PVDF Ultrafiltration Membrane with One Step Fabricated Zwitterionic Surface ACS Appl Mater Interfaces 7 1794717953

Zinadini S, Zinatizadeh A A, Rahimi M, Vatanpour V and Zangeneh H (2014) Preparation of a novel antifouling mixed matrix PES membrane by embedding graphene oxide nanoplates J Membr Sci 453 292-301

Zodrow K, Brunet L, Mahendra S, Li D, Zhang A, et al. (2009) Polysulfone ultrafiltration membranes impregnated with silver nanoparticles show improved biofouling resistance and virus removal Water Res $\mathbf{4 3}$ 715-723. 